\title{
Topological defects in crystals: A density-wave theory
}

\author{
M RAJ LAKSHMI, H R KRISHNA-MURTHY and \\ T V RAMAKRISHNAN \\ Department of Physics, Indian Institute of Science, Bangalore 560012 , India
}

\begin{abstract}
A new approach for describing dislocations and other topological defects in crystals, based on the density wave theory of Ramakrishnan and Yussouff is presented. Quantitative calculations are discussed in brief for the order parameter profiles, the atomic configuration and the free energy of a screw dislocation with Burgers vector $\vec{b}=(a / 2, a / 2$, $a / 2)$ in a bcc solid. Our results for the free energy of the dislocation in a crystal of size $R$, when expressed as $\left(\lambda b^{2} / 4 \pi\right) \ln (\alpha R /|b|)$ where $\lambda$ is the shear elastic constant, yield, for example, the value $x \simeq 1.85$ for sodium at its freezing temperature $\left(371^{\circ} \mathrm{K}\right)$. The density distribution in the presence of the dislocation shows that the dislocation core has a columnar character. To our knowledge, this study represents the first calculation of dislocation structure, including the core, within the framework of an order parameter theory incorporating thermal effects.
\end{abstract}

Keywords. Dislocations; core energy and structure; topological defects in crystals; density wave theory.

\section{Introduction}

In this paper we outline briefly (for details see Raj Lakshmi et al 1987) a new approach that we have developed for describing dislocations and other topological defects in crystals. This approach is an extension of the density-wave theory of Ramakrishnan and Yussouff (Ramakrishnan and Yussouff 1979; Haymet and Oxtoby 1981; Ramakrishnan 1984; for an uptodate list of references, see Haymet 1987) to allow for topological singularities in the phase of the density wave. It is analogous to theories for vortices in superfluids and superconductors (Fetter and Hohenberg 1969) in that it is an order parameter description and includes thermal effects.

\section{Description of inhomogeneous solids in the density wave theory}

In the density-wave theory of Ramakrishnan and Yussouff, the following functional gives the excess free energy for creating a general density configuration $\rho(r)$ in a liquid of uniform density $\rho_{l}$ :

$$
\begin{aligned}
& \beta\left(\Omega-\Omega_{l}\right)=\beta \int \mathrm{d} \mathbf{r} U_{e}(\mathbf{r})\left[\rho(\mathbf{r})-\rho_{l}\right]+\int \mathrm{d} \mathbf{r} \rho(\mathbf{r}) \ln \left[\rho(\mathbf{r}) / \rho_{l}\right] \\
& -\int \mathrm{d} \mathbf{r}\left[\rho(\mathbf{r})-\rho_{l}\right]-\frac{1}{2} \int \mathrm{d} \mathbf{r} \int \mathrm{d} \mathbf{r}_{1} c^{(2)}\left(\mathbf{r}, \mathbf{r}_{1}\right)\left[\rho(\mathbf{r})-\rho_{l}\right]\left[\rho\left(\mathbf{r}_{1}\right)-\rho_{l}\right]+\ldots
\end{aligned}
$$

where $U_{c}(\mathbf{r})$ is the external potential and $c^{(2)}(\mathbf{r})$ is the direct correlation function of the (supercooled) liquid; ( . . represents contributions from higher order correlations which we will neglect). 
Minimization of $\Omega$ gives the self-consistent equation:

$$
\rho(\mathbf{r})=\rho_{l} \exp \left[-\beta U_{e}(\mathbf{r})+\int \mathrm{d} \mathbf{r}_{1} c^{(2)}\left(\mathbf{r}, \mathbf{r}_{1}\right)\left[\rho\left(\mathbf{r}_{1}\right)-\rho_{l}\right]\right.
$$

As shown in the references above, even in the absence of $U_{e}(\mathbf{r})$, equation (2) can support non-uniform solutions for $\rho(\mathbf{r})$, with lower free energy than that of the uniform liquid provided $c^{(2)}(\mathbf{r})$ is sufficiently large; for example, for a uniform solid, one has a solution of the form

$$
\rho(\mathbf{r})=\rho_{l}\left[1+\sum_{G} \eta_{G}^{*} \exp (i \mathbf{G} \cdot \mathbf{r})\right]
$$

For an inhomogeneous solid, consider the circumstances where

$$
\rho(\mathbf{r})=\rho_{l}\left[1+\sum_{\mathbf{G}} \eta_{\mathbf{G}}(\mathbf{r}) \exp (i \mathbf{G} \cdot \mathbf{r})\right]
$$

such that $\left\{\eta_{G}(\mathbf{r})\right\}$ vary slowly on the scale of the unit cell and of the range of $c^{(2)}(\mathbf{r})$. Then, a gradient expansion plus a local approximation in (1) gives (Haymet and Oxtoby 1981)

Here

$$
\begin{aligned}
\frac{\beta\left(\Omega-\Omega_{l}\right)}{\rho_{l}}= & \int \operatorname{dr} \omega\left\{\eta_{G}(\mathbf{r})\right\}-\frac{1}{4} \int \operatorname{dr} c_{0}^{\prime \prime} \eta_{0}(\mathbf{r}) \nabla^{2} \eta_{0}(\mathbf{r}) \\
& -\frac{1}{4} \sum_{G \neq 0} \int \operatorname{dr} c_{G}^{\prime \prime} \eta_{-G}(\mathbf{r}) \frac{(G \cdot \nabla)^{2}}{|G|} \eta_{G}(\mathbf{r})+\ldots
\end{aligned}
$$

$$
\begin{aligned}
\omega\left\{\eta_{G}\right\}= & \frac{1}{v} \int^{\mathrm{cell}} \mathrm{d} \mathbf{r}\left[1+\sum_{G} \eta_{G} \exp (i \mathbf{G} \cdot \mathbf{r})\right] \ln \left[1+\sum_{G} \eta_{G} \exp (i \mathbf{G} \cdot \mathbf{r})\right] \\
& -\eta_{0}-\frac{1}{2} \sum_{G} c_{G}\left|\eta_{G}\right|^{2} .
\end{aligned}
$$

is the (local) free-energy functional of $\left\{\eta_{G}\right\}$ for the homogeneous case;

$$
c_{G} \equiv \rho_{l} \int \mathrm{d} \mathbf{r} \exp (i \mathbf{G} \cdot \mathbf{r}) c^{(2)}(\mathbf{r})=\left.\rho_{l} c^{(2)}(q)\right|_{q=G},\left.\quad c_{G}^{\prime \prime} \equiv \rho_{l} \frac{\mathrm{d}^{2} c^{(2)}(q)}{\mathrm{d} q^{2}}\right|_{q=G},
$$

and we have assumed $c_{G}^{\prime} \equiv \rho_{l} \mathrm{~d} c^{(2)}(q) /\left.\mathrm{d} q\right|_{q=G}=0$ for simplicity.

Minimizing (5) we get the self-consistent equations for $\left\{\eta_{G}(\mathbf{r})\right\}$ as

$$
\begin{aligned}
& \frac{1}{2} c_{0}^{\prime \prime} \nabla^{2} \eta_{0}(\mathbf{r})=\delta \omega / \delta \eta_{0} \equiv-U_{0}\left\{\eta_{0}(\mathbf{r}), \eta_{G}(\mathbf{r})\right\}, \\
& \frac{1}{2} c_{G}^{\prime \prime} \frac{(G . \nabla)^{2}}{|G|} \eta_{G}(\mathbf{r})=\delta \omega / \delta \eta_{G} \equiv-U_{-G}\left\{\eta_{0}(\mathbf{r}), \eta_{G}(\mathbf{r})\right\}
\end{aligned}
$$

Clearly, $U_{0}\left(\eta_{0},\left\{\eta_{G}\right\}\right) / \beta$ and $U_{G}\left(\eta_{0},\left\{\eta_{G}\right\}\right) / \beta$ can be interpreted as the Fourier components of an external potential that would be required to stabilize (locally) any given $\eta_{0}$ and $\left\{\eta_{G}\right\}$ in a homogeneous situation. They are hence determined as solutions of the implicit equation:

$$
1+\sum_{G} \eta_{G} \exp (i \mathbf{G} \cdot \mathbf{r})=\exp \left[\left(c_{0} \eta_{0}-U_{0}\right)+\sum_{G}\left(c_{G} \eta_{G}-U_{G}\right) \exp (i \mathbf{G} \cdot \mathbf{r})\right]
$$




\section{Application to dislocations}

In general, $\eta_{G}(\mathbf{r})$ are complex: $\left\{\eta_{G}(\mathbf{r})\right\}=\left\{\mu_{G}(\mathbf{r}) \exp \left(i \phi_{G}(\mathbf{r})\right\}\right.$. Dislocations are topological defects which are characterized by the condition $\oint \mathrm{d} \phi_{G}=2 \pi m$ for paths encircling the dislocation. Asymptotically, far from the core of the dislocation, one expects that the solid is locally periodic, i.e. $\nabla \phi_{G} \propto \mathbf{G} \Rightarrow \phi_{G}=\mathbf{G} \cdot \mathbf{u}(\mathbf{r}) ; \mathbf{u}(\mathbf{r})$ is then interpretable as the local displacement field in the solid. This condition is then equivalent to the more familiar one $\oint \mathrm{d} u=b$, where $b$ is the Burgers vector.

The singular part of the phase $\phi_{G}(\mathbf{r})$ is determined by the geometry and configuration of dislocations in terms of the singular part of the displacement field (Landau and Lifshitz 1970), for example (i) for a serew dislocation along $z$-axis with $\vec{b}=b \hat{z}, \vec{u}_{\text {sing }}(\mathbf{r})=b \hat{z} \phi / 2 \pi$, and (ii) for an edge dislocation along $z$-axis with $\vec{b}=b \hat{x}$, $\vec{u}_{\text {sing }}(\mathbf{r})=b \hat{x} \phi / 2 \pi$, where $\phi$ is the azimuthal angle.

Given these, the amplitudes $\left\{\mu_{G}(\mathbf{r})\right\}$ and the regular part of the phases $\left\{\phi_{G}(\mathbf{r})\right\}$ can be determined by solving the set of nonlinear differential equations (7), subject to the following boundary conditions

(i) as $|r| \rightarrow \infty,\left\{\mu_{G}(\mathbf{r})\right\} \rightarrow \eta_{G}^{*}$ of the undeformed solid,

(ii) as $|r| \rightarrow 0$, i.e., at the core of the dislocation, $\left\{\mu_{G}(\mathbf{r})\right\} \rightarrow 0$

for those $G$ for which $G \cdot b \neq 0$; that is, $\oint \mathrm{d} \phi_{G}=2 \pi m \neq 0$ as one goes around the dislocation.

\section{Specifics for a $\frac{1}{2}$ a [111] screw dislocation in a bec solid}

For simplicity, we take to be non-zero only $c_{0}\left(c_{G}\right.$ at $\left.G=0\right)$ and $c_{1}, c_{1} "\left(\equiv c_{G}, c_{G} "\right.$ evaluated at the first peak of $\left.c^{(2)}(q)\right)$. This approximation is known to give a reasonable description of the bec solid (Ramakrishnan and Yussouff 1979), its elastic properties (Ramakrishnan 1984) and its interface with the melt (Oxtoby and Haymet 1982).

Then, by the symmetry of the problem, only two independent amplitudes $\mu_{1}$ and $\mu_{2}$ are enough to characterize the dislocation. Namely, $\mu_{G}=\mu_{1}$ for $G=\{[110],[101]$, $[011]\}$ and $\{[\overline{1} 10],[\overline{10} \overline{1}],[0 \overline{1}]\}$ for which $\phi_{G}= \pm \phi$ respectively; and $\mu_{G}=\mu_{2}$ for $G=\{[1 \overline{10}],[10 \overline{1}],[01 \overline{1}],[\overline{1} 10],[\overline{101}],[0 \overline{1} 1]\}$ for which $\phi_{G}=0$. Furthermore, $\mu_{1}$ and $\mu_{2}$ will be functions only of the radial distance $r$ from the axis of the dislocation.

The set of nonlinear partial differential equations (7) now reduces to

$$
\begin{aligned}
& c_{1}^{\prime \prime}\left(\frac{\mathrm{d}^{2} \mu_{1}}{\mathrm{~d} r^{2}}+\frac{1 \mathrm{~d} \mu_{1}}{r}-\frac{\mu_{1}}{r^{2}}\right)=12 U_{1}\left(\mu_{1}, \mu_{2}\right), \\
& c_{1}^{\prime \prime}\left(\frac{\mathrm{d}^{2} \mu_{2}}{\mathrm{~d} r^{2}}+\frac{1 \mathrm{~d} \mu_{2}}{r}\right)=4 U_{2}\left(\mu_{1}, \mu_{2}\right) .
\end{aligned}
$$

Here, the functions $U_{1}$ and $U_{2}$ are determined as solutions of the following equations, which follow from (8):

$$
\begin{aligned}
& \left(\begin{array}{l}
\mu_{1} \\
\mu_{2}
\end{array}\right)=\frac{1}{\phi_{0}}\left(1+\frac{\ln \phi_{0}}{1-c_{0}}\right) \frac{1}{v} \int^{\text {cell }} \mathrm{d} \mathbf{r}\left(\begin{array}{l}
w_{1}(\mathbf{r}) \\
w_{2}(\mathbf{r})
\end{array}\right) \\
& \exp \left[6\left(c_{1} \mu_{1}-U_{1}\right) w_{1}(\mathbf{r})+6\left(c_{1} \mu_{2}-U_{2}\right) w_{2}(\mathbf{r})\right]
\end{aligned}
$$


where

$$
\begin{aligned}
& \phi_{0}=\frac{1}{v_{\text {cell }}} \int \mathrm{d} \mathbf{r} \exp \left[6\left(c_{1} \mu_{1}-U_{1}\right) w_{1}(\mathbf{r})+6\left(c_{1} \mu_{2}-U_{2}\right) w_{2}(\mathbf{r})\right], \\
& w_{1}(\mathbf{r})=\frac{1}{3}\left(\cos \frac{2 \pi}{a}(x+y)+\cos \frac{2 \pi}{a}(y+z)+\cos \frac{2 \pi}{a}(z+x)\right) \\
& w_{2}(\mathbf{r})=\frac{1}{3}\left(\cos \frac{2 \pi}{a}(x-y)+\cos \frac{2 \pi}{a}(y-z)+\cos \frac{2 \pi}{a}(z-x)\right)
\end{aligned}
$$

(In writing these equations, use has been made of the fact that $\left|c_{0}\right|$ is large, to make an expansion in powers of $\left.\left(1-c_{0}\right)^{-1}\right)$.

The coupled nonlinear differential equations (12) are to be solved with the following boundary conditions (cf., the previous section):

(i) as $r \rightarrow 0, \mu_{1} \rightarrow 0$ and $\mathrm{d} \mu_{2} / \mathrm{d} r \rightarrow 0$,

(ii) as $r \rightarrow \infty, \mu_{1}, \mu_{2} \rightarrow \eta_{1}{ }^{*}$.

( $\equiv \eta_{G}{ }^{*}$ for the first set of reciprocal lattice vectors for the uniform bcc solid.) (The amplitudes for the other reciprocal lattice vectors $G^{\prime}$ are determined from the equations $U_{G^{\prime}}\left(\mu_{1}, \mu_{2},\left\{\mu_{g^{\prime \prime}}\right\}\right)=0$.)

\section{Some details of the calculations}

The asymptotic behaviour of the solutions to (9) can be obtained analytically and is given by (with $\tilde{r}=r / \sqrt{\left|c_{1}{ }^{\prime \prime}\right|}$ )

(i) as $\tilde{r} \rightarrow 0$

$$
\begin{aligned}
& \mu_{1}=a_{1} \tilde{r}-4 U_{1}\left(0, a_{2}\right) \tilde{r}^{2}, \\
& \mu_{2}=a_{2}-U_{2}\left(0, a_{2}\right) \tilde{r}^{2},
\end{aligned}
$$

where $a_{1}, a_{2}$ are constants that are to be determined by solving the boundary value problem.

(ii) as $\tilde{r} \rightarrow \infty$

$$
\begin{aligned}
& \mu_{1}=\eta_{1}^{*}-\frac{\left(\partial U_{2} / \partial \mu_{2}\right)^{*} \eta_{1}^{*}}{\operatorname{det}} \cdot \frac{1}{\tilde{r}^{2}}+\ldots \\
& \mu_{2}=\eta_{1}^{*}-\frac{\left(\partial U_{2} / \partial \mu_{1}\right)^{*} \eta_{1}^{*}}{\operatorname{det}} \cdot \frac{1}{\tilde{r}^{2}}+\ldots
\end{aligned}
$$

where the ${ }^{*}$ on the derivatives means that these are to be evaluated for $\mu_{1}, \mu_{2}=\eta_{1}^{*}$ and

$$
\operatorname{det}=\left.\left(\frac{\partial U_{1}}{\partial \mu_{1}} \cdot \frac{\partial U_{2}}{\partial \mu_{2}}-\frac{\partial U_{1}}{\partial \mu_{2}} \cdot \frac{\partial U_{2}}{\partial \mu_{1}}\right)\right|_{\mu_{1}, \mu_{2}=\eta_{1}^{*}} .
$$

The $\tilde{r} \rightarrow \infty$ solution is consistent with what is expected for an elastically distorted solid with the displacement field $\vec{u}=\vec{b} \phi / 2 \pi$, and a shear elastic constant $\lambda=\rho_{l} k_{B} T\left|c_{1} "\right|\left|G_{1}\right|^{2} \eta_{1}^{* 2} / 3$ (Ramakrishnan 1984).

For obtaining the full numerical solution to (9), one has to know $U_{1}\left(\mu_{1}, \mu_{2}\right)$ and 
$U_{2}\left(\mu_{1}, \mu_{2}\right)$. The computation of these functions by solving (10) is extremely expensive in terms of computer time. However, we can use a trick to reduce the numerical work considerably. Instead of working with the variables $\mu_{1}, \mu_{2}$ we work with $\xi_{1}$, $\xi_{2}$ the fourier components of the molecular field, which are defined as

$$
\xi_{1} \equiv c_{1} \mu_{1}-U_{1} ; \xi_{2} \equiv c_{1} \mu_{2}-U_{2} .
$$

Then, using (10), $\mu_{1}, \mu_{2}$, and thence $U_{1}$ and $U_{2}$ are expressible as power series in $\xi_{1}$ and $\xi_{2}$, the coefficients of which series can be easily calculated. Equations (9) are now recast as (more complicated looking) differential equations for $\xi_{1}(r)$ and $\xi_{2}(r)$ and solved numerically. The asymptotic $r$-dependence of $\xi_{1}, \xi_{2}$ is similar to that of $\mu_{1}, \mu_{2}$ (for details see Raj Lakshmi et al 1987).

\section{Some results}

\subsection{Order-parameter profiles}

Figure 1 shows the profiles for $\xi_{1}(r), \xi_{2}(r)$ and $\eta_{0}(r)$, the fractional mean density

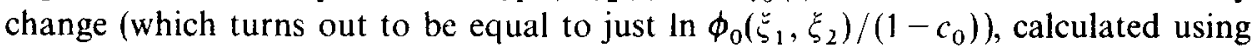
values for the parameters $c_{0}, c_{1}$ and $c_{1}$ " for sodium at its freezing temperature $T=371^{\circ} \mathrm{K} \equiv T_{f}$ (obtained from extrapolations of experimental data). This figure shows that the radius of the dislocation core is approximately 2-3 lattice spacings. Note also that $\xi_{1}(r)$ which characterizes $\xi_{G}(r)$ for those $G$ for which $\phi_{G} \neq 0$, vanishes at the core of the dislocation.

\subsection{Dislocation core eneryy}

Using the order parameter profiles we have calculated the free energy of the dislocation as the sum of two parts: (i) from the near region, using the numerical solution,

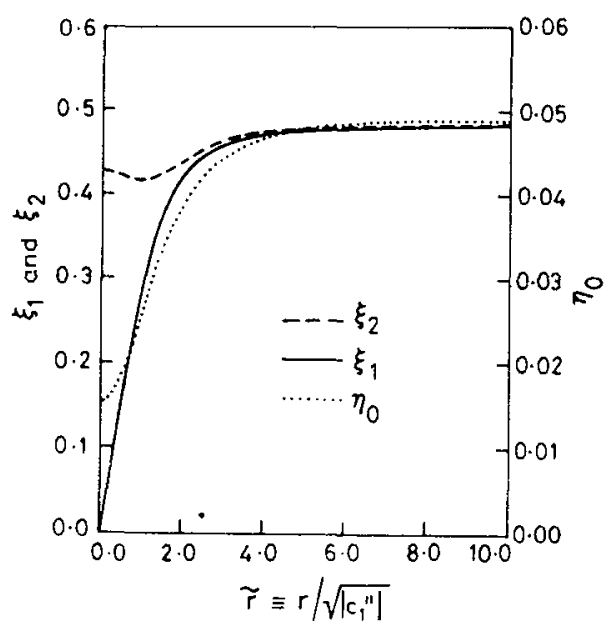

Figure 1. Profiles of the molecular field components $\xi_{1}(r)$ and $\xi_{2}(r)$, and the fractional

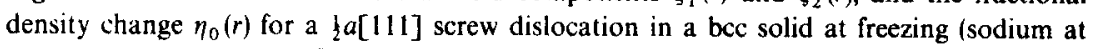
$T=T_{f}=371^{\prime} \mathrm{K}$ ). $\tilde{r}=r / \sqrt{\left|c_{1}{ }^{\prime \prime}\right|}$ is the reduced radial distance from the dislocation axis. 
and (ii) from the far region, using the asymptotic solution. It is conventional to express the result in the form $\left(\lambda b^{2} / 4 \pi\right) \ln (\alpha R / b)$, where $\lambda$ is the appropriate shear elastic constant. Then $\alpha$ is a measure of the core energy, and we get for $T=T_{f}\left(=371^{\circ} \mathrm{K}\right), \alpha=1.94 a / \sqrt{\mid c_{1}{ }^{\prime \prime}} \simeq 1.85$ (using experimental values for the parameters). We have also done the calculations for another temperature $T=T_{f}-100^{\circ}\left(=271^{\circ} \mathrm{K}\right)$ and find $\alpha=2.48 a / \sqrt{\mid c_{1}{ }^{\prime \prime}}$.

The only other calculation of the core energy is in the atomistic description (i.e. $T=0$ ) which gives $\alpha \sim 4-5$ (see Vitek et al 1970; Puls 1981; Christian 1983; Duesbery 1986).

\subsection{Configuration of the dislocation}

Within our formulation, we can obtain $\rho_{D}(r)$, the density distribution in the solid with the screw dislocation; this has the following features:

(i) $\rho_{D}(r)$ in the presence of the dislocation retains the same periodicity in the [111] direction as the uniform solid.

(ii) The positions of the maxima of $\rho_{D}(r)$, which may be identified with the atomic positions, are displaced in the presence of the dislocation, relative to the atomic positions in the uniform solid. These displacements are nearly the same as given by the continuum elasticity description, namely $\vec{v}=\vec{b} \phi / 2 \pi$, which is in the [111] direction. (iii) However, in terms of the full $\rho_{D}(r)$, the core region is greatly modified as compared to the uniform solid. Since $\xi_{1}(r)$ vanishes at the core of the dislocation, the modulation of $\rho_{D}(r)$ along the dislocation axis is small within the core. Thus the dislocation core has a columnar character.

\section{Concluding comments}

The scheme that we have presented here for describing dislocations in solids has the following advantages as compared to other schemes:

(i) Our scheme reduces to the correct elastic continuum theory far away from the dislocation. But the core region of the dislocation is adequately handled, unlike in the continuum elasticity theory.

(ii) Thermal, statistical mechanical effects are included from the outset, whereas the atomistic description is essentially a zero-temperature theory.

(iii) The parameters which are inputs to the theory are structural properties of the (supercooled) liquid which are either directly measurable or can be related to measured quantities. In contrast, the atomistic theory uses poorly known atomic potentials.

At the level of approximation discussed here, the free energy of the dislocation is independent of its location in the unit cell. This implies a zero Peierls-Nabarro barrier, i.e. the dislocation can move freely through the crystal. The degree of validity of the gradient and local approximations depends on the largeness of $c_{1}{ }^{\prime \prime}$. In our theory, the smallness of the experimentally observed barrier $\left(\sim 10^{-6}\right.$ of the extrapolated elastic energy needed for $10 \%$ strain, say) is understandable (and calculable) in terms of the small parameter $1 /\left|c_{1}{ }^{\prime \prime}\right|$ by going beyond the gradient and local approximations. In addition, our calculations can be improved to include more of the detailed $q$-dependence of $c^{(2)}(q)$ (which means keeping more fourier components of the molecular field). In that case, they can provide a valuable 
framework for investigating the structure and energetics of dislocations and other topological defects (such as grain boundaries) in crystals.

\section{References}

Christian J W 1983 Metall. Trans. A14 123

Duesbery M S 1986 Contemp. Phys. 27145

Fetter A L and Hohenberg P C 1969 Superconductivity (ed.) R D Parks (New York: M. Dekker), Vol. 2 Haymet A D J 1987 Ann. Rev. Phys. Chem. (submitted).

Haymet A D J and Oxtoby D W 1982 J. Chem. Phys. 742559

Landau L and Lifshitz L D 1970 Theory of elasticity 2nd ed. (Oxford: Pergamon).

Oxtoby D W and Haymet A D J 1982 J. Chem. Phys. 766262

Puls M P 1981 in Proc. Int. Conf. on Dislocation Modelling of Physical Systems (Oxford: Pergamon) p. 249

Raj Lakshmi M, Krishnamurthy H R and Ramakrishnan T V 1987 Phys. Rev. B (submitted).

Ramakrishnan T V 1984 Pramana-J. Phys. 22365

Ramakrishnan T V and Yussouff M 1979 Phys. Rev. B19 2775

Vitek V, Perrin R C and Bowen D K 1970 Philos. Mag. 211049 